\title{
Análise do comportamento da rede LoraWAN pública da cidade de Caxias do Sul/RS
}

\section{Analysis of the behavior of the public LoraWAN network in a city in southern Brazil}

\author{
Samuel Francisco Ferrigo ${ }^{\circledR}, 1$ and Joel da Silva ${ }^{1}$ \\ ${ }^{1}$ Centro Universitário Uniftec \\ *samuelferrigo@acad.ftec.com.br; joel_387@acad.ftec.com.br
}

Recebido: 12/03/2021. Revisado: 15/06/2021. Aceito: 28/06/2021.

\begin{abstract}
Resumo
O LoraWAN é uma rede de longa distância com baixo consumo energético utilizada globalmente na comunicação da Internet das Coisas. Neste trabalho é avaliada a confiabilidade e o alcance da rede LoraWAN pública instalada na cidade de Caxias do Sul/RS, realizando testes em diferentes locais e com alterações no fator de espalhamento e no tamanho do payload. Como resultados obtidos, verificou-se que a localização e a variação do fator de espalhamento interferiram na taxa de recebimento de quadros, enquanto o tamanho do payload não impacta nesse percentual.
\end{abstract}

Palavras-Chave: Confiabilidade, LoRa, LoraWAN, Taxa de recebimento de quadros.

\begin{abstract}
LoraWAN is a low-power wide area network used globally in Internet of Things communication. This work evaluates the reliability and reach of the public LoraWAN network installed in the city of Caxias do Sul/RS, performing tests in different locations and making changes in spreading factor and payload size. As results obtained, location and variation of the spreading factor interfered with the rate of receiving frames, while the payload size does not impact this rate.
\end{abstract}

Keywords: Frame receiving datarate, LoRa, LoraWAN, Reliability.

\section{Introdução}

No final do milênio passado, as redes de comunicação de dados eram classificadas em três grupos: redes locais (LANs), redes metropolitanas (MANs) e redes de longa distância (WANs) (Tanenbaum, 2003). Todavia, com o surgimento das redes de sensores sem fio e sua renomeação para Internet das Coisas (IoT), sensores, atuadores, dispositivos de smart grids e outras "coisas" mais (Souza et al., 2019, Noura et al., 2020) passaram a ser conectados a mesma Internet que já interligava computadores, notebooks e smartphones.

Como consequência, houve um aumento exponencial na quantidade de dispositivos conectados à rede. Somente em 2019, esse número chegou a 4,8 bilhões (Gartner, 2019). Assim sendo, essa divisão precisou ser ampliada para comportar as especifidades emergentes. Surgiram as redes pessoais (PANs), as redes corporais (BANs) (Ferrigo et al., 2017) e as redes de longa distância com baixo consumo energético (LPWANs).

Diferentemente das demais tecnologias de WAN sem fio, como WiMAX, 3G e 4G, as LPWANs são redes que, além de permitir a comunicação de longa distância, possuem a característica de baixo consumo de energia. Estas redes têm por finalidade atender aplicações de comunicação máquina a máquina (M2M) e IoT em geral (Meireles et al., 
2018), especialmente a dispositivos que possuem acesso limitado a fontes de energia.

As principais tecnologias LPWAN utilizadas atualmente são todas proprietárias: Narrowband IoT (NB-IOT), SigFox e Long Range (LoRa) (Thieme, 2020). Esta última, porém, possui uma comunidade global com mais de 100 mil membros e dispõe da associação LoRa Alliance, constituída pelas maiores empresas do mercado de IoT, o que corrobora para agregar funcionalidades, contribui para garantir a interoperabilidade da tecnologia e fez surgir a rede LoraWAN (Network, 2020c).

No município de Caxias do Sul/RS, essa comunidade é apoiada principalmente por uma associação de empresas de informática da região, conhecida por Trino Polo (Polo, 2020). Em 2018, essa entidade adquiriu e instalou gateways públicos LoRa em três pontos da zona urbana da cidade, inserindo-a no mapa mundial da IoT (Pioneiro, 2018).

Dessa forma, o objetivo desse trabalho é a avaliação dessa rede pública LoraWAN em relação à confiabilidade e ao alcance dessa rede, especialmente em relação à taxa de transmissão de quadros LoraWAN. Esta avaliação contemplou diversos locais, cada qual com diferentes características geográficas, bem como promoveu alterações de parâmetros que interferem no funcionamento da rede, dentre os quais a carga de dados (payload) enviada por cada quadro e o fator de espalhamento (SF).

O artigo está estruturado da seguinte forma: a Seção 2 detalha o funcionamento da comunicação LoRa e dos protocolos que formam a pilha LoraWAN utilizados para a comunicação de dispositivos LoRa. A Seção 3 explica o funcionamento da estrutura que recebe os dados de dispositivos LoRa. A Seção 4 discute os trabalhos que já avaliaram a tecnologia LoRa/LoraWAN no Brasil. A Seção 5 apresenta o ambiente de avaliação da tecnologia, enquanto a Seção 6 apresenta e discute os resultados obtidos.

\section{A pilha de protocolos LoraWAN}

A pilha de protocolos LoraWAN, mostrada na Fig. 1, é formada pela camada física LoRa, uma subcamada de acesso ao meio (MAC) e pela camada de aplicação. Esta última representa os dados de uma aplicação LoraWAN ou os dados de um gateway que permite a comunicação dessa tecnologia com o padrão TCP/IP através da arquitetura detalhada na Seção 3.

\subsection{A camada física LoRa}

Proprietária da Semtech, a camada física LoRa é responsável pelos parâmetros físicos de comunicação entre os dispositivos e sua de modulação do sinal não é totalmente aberta (Augustin et al., 2016). Conforme o país, pode operar em diferentes faixas sub-Ghz não licenciadas, chamadas de bandas industriais, científicas e médicas (ISM). Estas faixas utilizam frequências de $430 \mathrm{MHz}, 868 \mathrm{MHz}$ e $915 \mathrm{MHz}$. Cada uma dessas faixas faixa possui vários canais para transmissão de dados (LoRa, 2017).

O LoRa utiliza técnica de modulação baseada na Chirp Spread Spectrum (CSS), permitindo que os dados possam ser transmitidos com baixos níveis de potência e menos

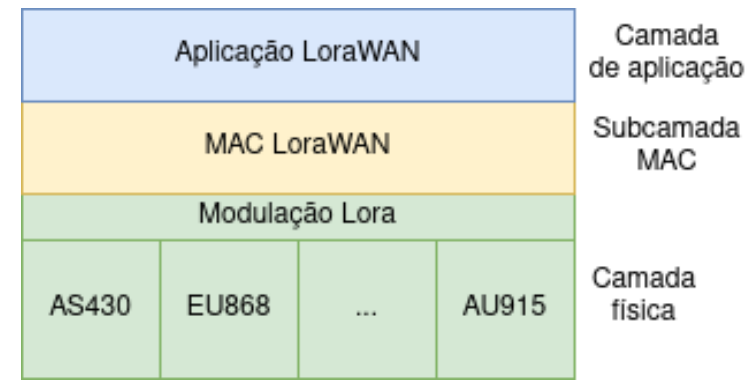

Figura 1: Estrutura da arquitetura LoraWAN. Fonte: Elaborada pelo autor.

suscetíveis a ruídos de transmissão (Villarim et al., 2019). A modulação LoRa permite parametrizações de fator de espalhamento (SF), largura de banda (BW) e taxa de código (CR). Essas parametrizações definem a taxa de transmissão (TR) em bits do canal, através da seguinte fórmula (Kufakunesu et al., 2020):

$$
\mathrm{TR}=S F \frac{B W}{2^{S F}} C R
$$

A BW define quantos chirps podem ser enviados por segundo (Augustin et al., 2016). Cada hertz da BW corresponde a um chirp. Portanto, quanto maior a BW, maior será a quantidade de chirps e, consequentemente, maior será taxa de transmissão do canal.

O SF define a duração de cada chirp. Quanto maior for o SF, maior a duração do chirp e, portanto, menor a taxa de transmissão do canal. Consequentemente, quanto maior for o SF, maior será o consumo energético, porém maior será a distância que poderá ser alcançada pelo chirp $(\mathrm{Au}-$ gustin et al., 2016). Já o CR permite a correção de erros na transmissão dos dados. Quanto menor o CR, menor será o overhead de correção dos eventuais erros na recepção (Benkahla et al., 2021).

O LoRa permite que esses parâmetros (BW, SF e CR) sejam definidos de maneira manual ou dinâmica, através da tecnologia de Taxa de Dados Adaptativa (ADR). Caso o usuário opte pelo uso da ADR, a potência de transmissão também é definida automaticamente (Meireles et al., 2018).

No Brasil esses parâmetros são definidos pela Anatel (Network, 2020b). A faixa permitida e mais usual para emprego do LoRa compreende o intervalo de 915 a 928 $\mathrm{MHz}$ (Chinato et al., 2020). Esta faixa, denominada AU915928, se divide em 8 sub-bandas, perfazendo um total de 64 canais uplink de $125 \mathrm{kHz}, 8$ canais uplink de $500 \mathrm{kHz}$ e 8 canais downlink de $500 \mathrm{kHz}$.

Apesar da referida disponibilidade de canais, os dispositivos LoRa são configurados para utilizar-se de apenas uma sub-banda, composta de 8 canais de $125 \mathrm{kHz}$ cada para uplink, 1 canal de $500 \mathrm{kHz}$ também para uplink, e 8 canais de $500 \mathrm{kHz}$ para downlink. As configurações de SF permitidas são de 7 a 10 para uplink e 7 a 12 para downlink (Network, 2020a).

Essa camada também prevê um formato de transmissão dos dados, composto por um preâmbulo, um payload com- 
posto pelo quadro da subcamada MAC e, em mensagens de uplink, por um checksum (CRC) que verifica a integridade dos dados recebidos (LoRa, 2017).

\subsection{A subcamada de acesso ao meio (MAC)}

Acima da camada física encontra-se a subcamada de acesso ao meio. Essa camada é responsável por realizar o enquadramento dos dados recebidos da camada de aplicação e entregá-los a outros dispositivos LoRa que estejam na mesma rede - no caso de redes LoRa "puras" - ou para o gateway que enviará os dados para a LoraWAN. Esta camada também implementa um protocolo baseado no ALOHA para evitar colisões na transmissão, com a função de reduzir a complexidade dos dispositivos e apresentando um desempenho superior à versão original (Silva et al., 2021).

No caso de redes LoraWAN, os dados são enviados através de quadros que possuem a estrutura básica mostrada na Fig. 2. Os campos DevAddr, FCnt, FOpts e FPort são os principais campos que formam o cabeçalho MAC (Augustin et al., 2016). Outros campos podem ser adicionados ao cabeçalho, dependendo da necessidade. Por esse motivo, o tamanho do cabeçalho pode variar entre 13 e 28 bytes. O campo DevAddr contém o endereço do dispositivo LoRaWAN. FCtrl possui diversas parametrizações, como solicitar a retransmissão de quadros e utilizar a taxa de dados adaptativa (ADR) (LoRa, 2017), explicada na Seção 2.1.

Seguindo na estrutura do quadro MAC, FCnt é o contador de quadro, permitindo que o receptor possa reconstruir os dados e, caso habilitado em FCtrl, solicite a retransmissão do quadro ao transmissor (Ertürk et al., 2019). FOpts permite o envio de comandos MAC. FPort indica a porta de recebimento, fornecendo o uso de multiplexação. O campo payload é o espaço destinado para envio dos dados criptografados da aplicação LoRa. Esse espaço é de, no máximo, 51 bytes quando utilizados canais de $125 \mathrm{kHz}$. Os dados são criptografados através do padrão AES 128 bits (LoRa, 2017).

Essa subcamada também é responsável por dividir os dispositivos em três classes identificadas pelas letras A, B e $\mathrm{C}$, de acordo com a necessidade de cada aplicação LoRa. Dispositivos de classe A permitem o agendamento do uplink dos dados de acordo com sua necessidade. Após a transmissão dos dados, abrem-se duas curtas janelas de downlink onde esses dispositivos podem receber dados (Triantafyllou et al., 2021). No restante do tempo o transceptor desses dispositivos permanecem em estado de dormência. Dessa forma, dispositivos de classe A possuem maior eficiência energética, porém possuem menos flexibilidade para receber dados (Augustin et al., 2016).

Dispositivos de classe $B$ funcionam de maneira análoga aos dispositivos de classe $\mathrm{A}$, porém podem ser programados com maior número de janelas de downlink de recepção

\begin{tabular}{|l|l|l|l|l|l|}
\hline DevAddr & FCtrl & FCnt & FOpts & FPort & Payload \\
\hline
\end{tabular}

Figura 2: Estrutura básica do quadro MAC LoRaWAN. Fonte: Elaborada pelo autor. de dados. Já dispositivos de classe $\mathrm{C}$ possuem intervalo contínuo de envio e recebimento de dados, ou seja, o rádio permanece sempre ativo. Assim, são os que mais consomem energia elétrica.

\section{A arquitetura LoraWAN}

Além dos dispositivos LoRa, a arquitetura LoraWAN é composta por gateways, servidores de rede (network servers) e servidores de aplicação (application servers), formando uma topologia chamada de estrela-das-estrelas (Chinchilla-Romero et al., 2021), conforme Fig. 3. Gateways recebem os dados dos dispositivos LoraWAN transmitidos por meio do protocolo LoRa descritos na Seção 2. Como pode ser observado na Fig. 2, dispositivos LoRa não podem comunicar-se diretamente com outros dispositivos LoRa em redes LoraWAN, uma vez que o cabeçalho MAC comporta somente o endereço do dispositivos de origem dos dados. Assim sendo, só os gateways LoraWAN situados no raio de cobertura de um dispositivo são capazes de receber os dados difundidos pelo dispositivo.

Outra importante função desses dispositivos é converter os dados recebidos em formato LoraWAN para o padrão $\mathrm{TCP} / \mathrm{IP}$. Isso permite que os dados sejam transmitidos na Internet para os servidores de rede. Essa transmissão é feita através do protocolo Message Queue Transfer Transport (MQTT), através do método de publish/subscribe (Barro et al., 2019). Nesse método, apenas os servidores de rede assinantes recebem os dados publicados pelos dispositivos LoRa registrados na rede LoraWAN.

Esses servidores gerenciam a rede LoraWAN (Noura et al., 2020), sendo responsáveis por descriptografar os dados recebidos dos dispositivos e entregá-los aos servidores de aplicação, bem como realizar o processo inverso: criptografar os dados das aplicações e enviá-los aos dispositivos (Augustin et al., 2016). Os servidores de rede também são responsáveis por armazenar as informações dos gateways e dos dispositivos registrados na rede LoraWAN. A comunicação entre os servidores de rede e os servidores de aplicação ocorrem através de Interfaces de Programação de Aplicativos (APIs) desenvolvidos para essa finalidade.

Além disso, caso mais de um gateway receba dados enviados de um mesmo dispositivo, os servidores de rede são responsáveis por descartar as réplicas, bem como também se encarrega de avaliar, dentre os gateways, aquele em melhor condições de efetuar o downlink, caso necessário.

Dessa forma, os servidores de rede exercem um papel central na comunicação da rede LoraWAN, pois estes detêm os endereços dos gateways e dos dispositivos. Consequentemente, os dados são enviados sempre de um ou vários dispositivos para um ou vários servidores de aplicação, e vice-versa.

\section{Trabalhos relacionados}

Os primeiros trabalhos envolvendo o uso tecnologia LoraWAN no Brasil datam do ano de 2015 no trabalho de Marca and Scholze (2015), que propôs a substituição das tecnologias de comunicação GSM por LoraWAN em smart grids. Foram realizados experimentos analisando o indica- 


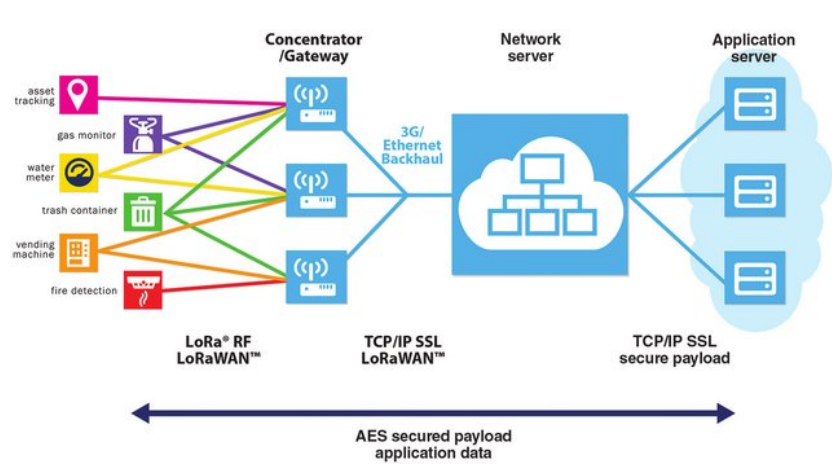

Figura 3: Estrutura LoraWAN. Fonte: Retirado de Network (2020c).

dor de intensidade do sinal recebido (RSSI) em diferentes distâncias.

Soares E Silva et al. (2018), ao contrário dos demais trabalhos, propôs o uso de redes LoraWAN em áreas rurais, onde as redes de telefonia móvel não estão disponíveis. Como resultados preliminares, montou-se uma rede LoraWAN no campus da Universidade Federal de Santa Maria/RS, onde foram analisadas as variações de RSSI a partir do uso de antenas com diferentes ganhos.

O estudo de Meireles et al. (2018) apresentou os resultados de uma rede LoraWAN experimental implantada na cidade de Ouro Preto/MG, que é caracterizada por estar situada em uma região montanhosa. Foram realizados testes que avaliaram a taxa de recepção de quadros e o valor de RSSI em diferentes lugares.

Ortiz et al. (2018), em seu trabalho, realizou testes sobre a tecnologia LoRa no campus da Universidade Federal do Rio de Janeiro, realizando modificações no SF. Foram medidas as taxas de RSSI em diferentes lugares do campus. Neste trabalho não é utilizada a estrutura LoraWAN.

Villarim et al. (2019), de maneira análoga ao trabalho anterior, avalia o uso somente da LoRa, mas na cidade de João Pessoa/PB. Nele realizou análises em dois cenários distintos: numa área com alta densidade de prédios e numa floresta urbana. Nos testes realizados foram medidos a variação do RSSI conforme a distância, e os resultados entre os cenários foram comparados.

Finalmente, Ferrari et al. (2020) propõe o uso da tecnologia LoraWAN como alternativa ao uso de redes $3 \mathrm{G} / 4 \mathrm{G}$ no monitoramento de veículos em cidades inteligentes. Como cenário de avaliação, um gateway LoraWAN foi instalado no campus da Universidade Federal do Rio Grande do Norte, e verificado a cobertura do sinal em 20 locais da região.

Todos os trabalhos anteriormente relacionados tiveram seus experimentos aplicados sobre um ambiente controlado, visto que a estrutura LoraWAN não existia previamente. Este trabalho, em compensação, explora essa lacuna: avalia o uso da tecnologia LoraWAN em uma rede pública e em uso há cerca de dois anos. A avaliação dessa rede consistiu em analisar o seu alcance e a sua confiabilidade, através da medição da taxa de recepção de quadros em diferentes locais da cidade de Caxias do Sul/RS, cada qual com suas peculiaridades, e utilizando variações no SF e no tamanho do payload do quadro.

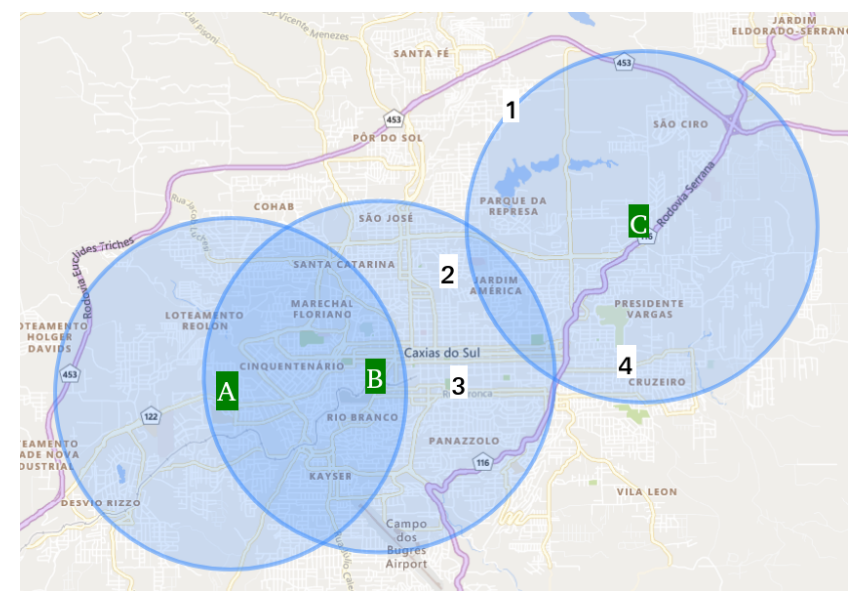

Figura 4: Estimativa de cobertura de cada um dos gateways públicos (identificados por letras) e dos dispositivos (identificados por números). Fonte: Elaborada pelo autor.

\section{Ambiente de avaliação}

O ambiente de avaliação foi executado na cidade de Caxias do Sul, localizado na serra geral do estado do Rio Grande do Sul. Possui uma população de cerca de 517 mil habitantes (IBGE, 2020), sua área central possui grande quantidade de edifícios e está localizada a uma altitude de 760 metros (Caxias do Sul, 2014). Em diferentes locais dessa cidade foi posicionado um dispositivo Heltec LoRa ${ }^{1}$, um hardware voltado ao desenvolvimento de aplicações IoT baseado no microcontrolador ESP32, cujas características gerais são mostradas na Tabela 1. Esse dispositivo esteve ligado por, no mínimo, três dias em cada um dos locais identificados nas Figs. 4 e 5 pelos números 1, 2, 3 e 4.

\begin{tabular}{cc} 
Tabela 1: Características do dispositivo Lora. \\
Característica & Descrição \\
\hline Processador & 240MHz dual-core \\
RAM & $520 \mathrm{~KB}$ \\
Potência de transmissão & $18 \mathrm{~dB}$ \\
Antena & 3dBi Omnidirecional \\
Lora Chip & SX1276 \\
Interfaces de comunicação adicionais & Wi-Fi/Bluetooth \\
\hline
\end{tabular}

O local 1 está na região norte da cidade, numa altitude bastante superior (828 metros) à altitude da cidade e com baixa densidade de edifícios. O local 2 está na região central, numa altitude também superior (794 metros) à altitude da cidade e com média densidade de prédios. Já o local 3 também está na região central, numa altitude próxima à da cidade (768 metros) e com alta densidade de edifícios. Por fim, o local 4 está na região leste, num vale, a uma altitude inferior (749 metros) à da cidade e com média densidade de edifícios. Em todos os locais os testes foram

1 https://heltec.org/project/wifi-lora-32/ 


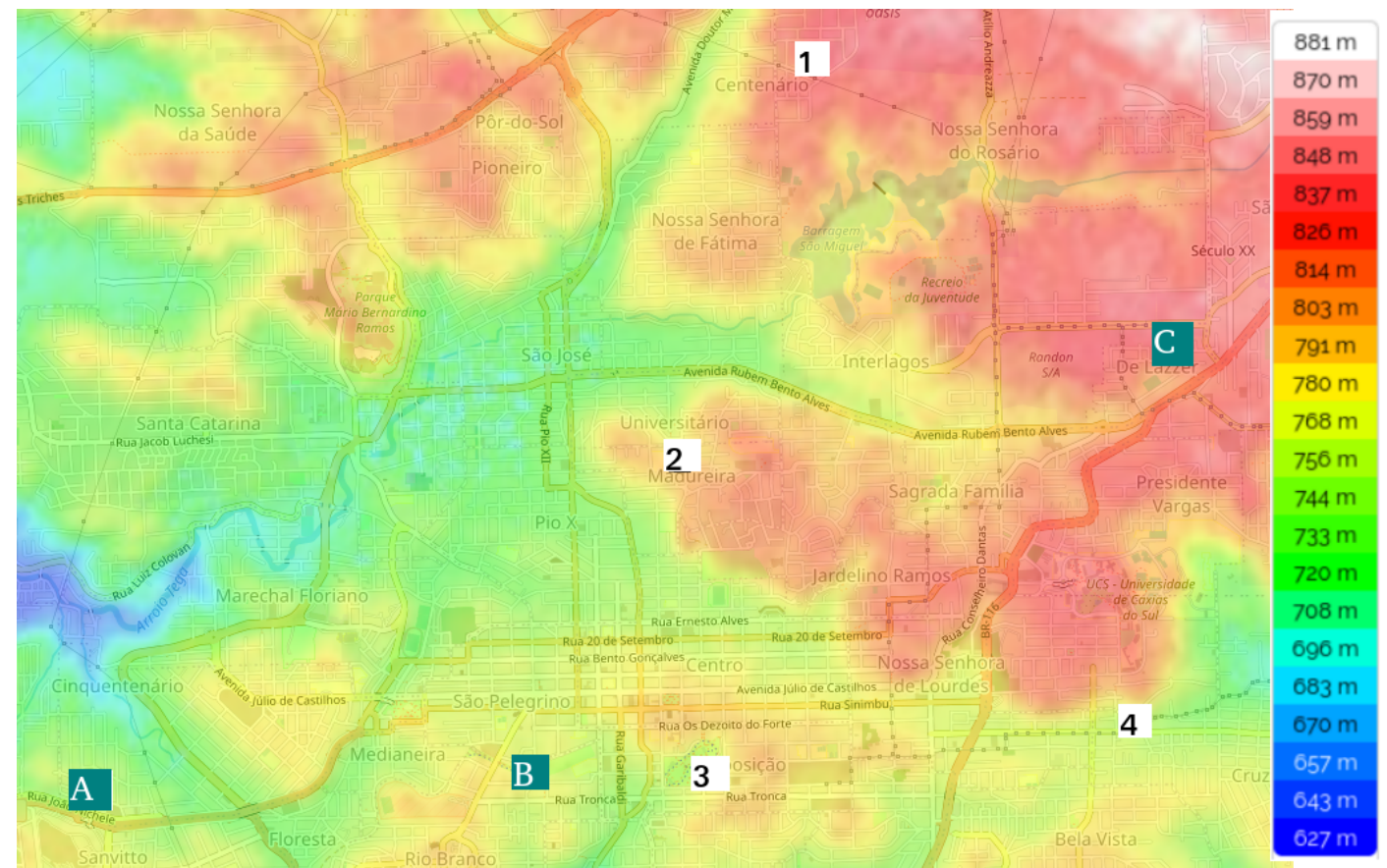

Figura 5: Representação em altitude do mapa urbano de Caxias do Sul/RS com a localização dos gateways públicos LoraWAN (identificados por letras) e dos dispositivos (identificados por números). Fonte: Elaborada pelo autor.

executados em ambientes indoor.

O dispositivo foi configurado para comunicar-se com os gateways públicos LoraWAN instalados pelo Trino Polo por meio da biblioteca LMIC $^{2}$. A configuração do dispositivo previa o envio de um quadro a cada 30 segundos, sendo que a cada envio era modificado sequencialmente o canal de transmissão ( 0 a 7, conforme Tabela 2) através da técnica de round-robin. Os gateways estão identificados nas Figs. 4 e 5 pelas letras $\mathrm{A}, \mathrm{B}$ e $\mathrm{C}$.

O gateway A está instalado no prédio sede do Centro Universitário UniFTEC, localizado na zona oeste da cidade. O local está numa altitude próxima $(757 \mathrm{~m})$ à da cidade $\mathrm{e}$ possui uma densidade média de prédios. Já o gateway $\mathrm{B}$ está instalado na região central da cidade, acima da altitude da cidade $(780 \mathrm{~m})$ e possui uma alta densidade de prédios. Já o gateway C está instalado na zona nordeste da cidade, numa altitude muito superior à da cidade $(833 \mathrm{~m})$ e possui uma densidade baixa de edifícios.

Além disso, segundo estimativa mostrada na comunidade da The Things Network de Caxias do Sul ${ }^{3}$, todos os locais estão cobertos pelo sinal de algum dos gateways públicos do Trino Polo, conforme mostra a Fig. 4. Essa estimativa considera que um gateway é capaz de atender um raio de aproximadamente $3 \mathrm{~km}$.

Por questões desconhecidas até a escrita deste trabalho, os gateways públicos estavam configurados para a banda ISM US902-928, ao invés da faixa AU915-928 homologadas pela Anatel. Todas as especificações entre as bandas seguem os parâmetros descritos na Seção 2.1, exceto as

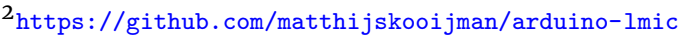

3https://www.thethingsnetwork.org/community/caxias-do-sul/
}

frequências dos canais de uplink, que variam de uma banda para outra (Network, 2020a), conforme Tabela 2.

Tabela 2: Frequências de operação, em $\mathrm{MHz}$, dos canais

\begin{tabular}{ccc}
\multicolumn{3}{c}{ uplink } \\
Canal & US902-928 & AU915-928 \\
\hline 0 & 903.9 & 916.8 \\
1 & 904.1 & 917.0 \\
2 & 904.3 & 917.2 \\
3 & 904.5 & 917.4 \\
4 & 904.7 & 917.6 \\
5 & 904.9 & 917.8 \\
6 & 905.1 & 918.0 \\
7 & 905.3 & 918.2 \\
\hline
\end{tabular}

Dessa forma, procurou-se analisar a transmissão de dados nessa rede pública considerando o uso do mesmo hardware e considerando o cenário mais heterogêneo possível dentro da previsão de cobertura de sinal LoraWAN mostrada na Fig. 4. Essa heterogeneidade era composta por variações de altitude, densidade de edifícios, distância entre dispositivos e gateways e uso de diferentes canais LoraWAN. A distância entre os locais e os gateways são apresentados na Tabela 3.

O servidor de aplicação foi criado com a finalidade armazenar os dados enviados pelo dispositivo e encaminhado pelos gateways ao servidor de rede. Além dos dados do dispositivo, os gateways enviam dados adicionais, como informações do gateway, relação sinal/ruído (SNR), RSSI, canal, BW, SF e frequência do canal, horário de recebimento do 
quadro, bem como informações do próprio quadro MAC.

O servidor de rede, por sua vez, encaminhava os dados recebidos, estruturados no formato JSON, através de uma API de integração que utiliza o protocolo HTTP. Os dados recebidos foram armazenados pela aplicação num banco de dados MySQL.

Tabela 3: Distâncias entre nós e gateways. Entre parênteses consta a altitude de cada local e gateway.

\begin{tabular}{cccc} 
Local/Gateway & $\mathrm{A}(757 \mathrm{~m})$ & $\mathrm{B}(78 \mathrm{om})$ & $\mathrm{C}(833 \mathrm{~m})$ \\
\hline Local 1(828m) & $6,68 \mathrm{~km}$ & $4,94 \mathrm{~km}$ & $3,06 \mathrm{~km}$ \\
Local 2 (794m) & $4,43 \mathrm{~km}$ & $2,30 \mathrm{~km}$ & $3,04 \mathrm{~km}$ \\
Local 3 (768m) & $3,95 \mathrm{~km}$ & $1,35 \mathrm{~km}$ & $4,29 \mathrm{~km}$ \\
Local 4 (749m) & $6,76 \mathrm{~km}$ & $4,13 \mathrm{~km}$ & $2,47 \mathrm{~km}$ \\
\hline
\end{tabular}

\subsection{Testes realizados}

Para avaliação do comportamento dessa rede, foram realizados dois tipos de testes, todos eles realizados utilizando a banda ISM US902-928 configurados nos gateways referenciados na Seção 5. O primeiro consiste em analisar a transmissão de quadros a partir de diferentes locais, considerando o envio de 20 bytes de dados a cada comunicação. A escolha por 20 bytes foi definida de forma a considerar que o dispositivo enviasse dados de temperatura e umidade do local. Esse teste foi realizado nos locais 1 a 4 utilizando, em cada local, SF 7 e 10, que são, respectivamente, os valores mínimo e máximo permitidos pela banda configurada nos gateways. Portanto, cada teste consistia em avaliar o impacto da variação extrema do SF na confiabilidade da comunicação em cada local. Em cada um desses testes foram realizados 200 envios.

Já o segundo teste foi realizado somente no local 2, e consistiu em analisar o impacto do tamanho do payload no envio dos dados para os diferentes gateways, utilizando todos os SF permitidos pela banda. Os testes foram executados em payloads com carga de 1, 12, 25 e 51 bytes - 0 limite do quadro, conforme descrito na Seção 2.2. Dessa forma, pretendeu-se analisar o impacto do tamanho do payload, bem como o impacto da variação do SF na confiabilidade da comunicação entre o dispositivo e os gateways. Em cada um desses testes foram realizados 100 envios.

\section{Resultados e discussões}

Os resultados e discussões dos testes efetuados estão divididos em duas categorias, detalhadas nas Seções a seguir.

\subsection{Avaliação conforme a mudança de local do dis- positivo LoraWAN}

Conforme mostrado na Fig. 4, era esperado que o dispositivo instalado no local 1 conseguisse comunicar-se apenas com o gateway C. Todavia, conforme apresentado na Fig. 6, todos os gateways conseguiram comunicar-se com o dispositivo. Como pode ser observado, neste local, houve uma concentração de recebimento dos quadros no gateway C, por ser o gateway mais próximo desse local.

Utilizando SF10, a taxa de recebimento dos quadros variou entre $43 \%$ e $80 \%$ nos gateways. Considerando o somatório dos três gateways, a taxa de recebimento ficou em $81,5 \%$, um pouco acima do percentual recebido pelo gateway $\mathrm{C}$. Isso ocorre porque, em muitos envios, o quadro é recepcionado por mais de um gateway.

Um detalhe observado - e que se repete nas demais avaliações - é de que o gateway B está configurado apenas para uso dos canais 0 a 3. Isso somente foi percebido ao analisar os quadros recebidos, onde verificou-se que em nenhum dos casos a taxa de recebimento ultrapassou $50 \%$. Isso deve-se ao fato de que o dispositivo envia os quadros de forma aleatória por todos os canais da banda US902-928, conforme explicado na Seção 5. Essa característica explica o motivo pelo qual, neste caso, a taxa de recebimento de quadros do gateway $\mathrm{A}$ foi superior à $\mathrm{B}$, apesar de estar mais distante do dispositivo.

Ao alterar a configuração do dispositivo para SF7, há uma redução da taxa de recebimento total para $53 \%$, sendo que o gateway Aé o que sofre proporcionalmente a redução mais drástica, de $48,5 \%$ para $10 \%$, enquanto o gateway $\mathrm{C}$, por ser o mais próximo, é proporcionalmente o menos afetado, com redução de $80 \%$ para $49,5 \%$. Esses dados também são demonstrados na Fig. 6.

Em relação ao local 2, havia a expectativa de comunicação apenas com o gateway B, conforme Fig. 4. Todavia, também neste caso, o dispositivo consegue comunicar-se com todos os gateways, havendo, inclusive, uma distribuição homogênea no recebimento de quadros entre esses equipamentos, conforme apresentado na Fig. 7. Utilizando SF10, a taxa de recebimento dos quadros variou entre $26 \%$ e $38 \%$ nos gateways. Considerando o somatório dos três, a taxa de recebimento ficou em $68 \%$. Percebe-se, neste caso, que na maior parte dos envios, apenas um gateway recebia o quadro.

Ao alterar a configuração do dispositivo para SF7, há uma redução da taxa de recebimento total para $14 \%$, sendo que praticamente todos são recebidos pelo gateway $\mathrm{B}$, conforme também demonstrado na Fig. 7. Dessa forma, verifica-se que os gateways A e C são drasticamente afetados com a mudança do SF, enquanto B é o menos afetado. Além disso, caso o este último estivesse configurado para uso de todos os canais, possivelmente a taxa de recepção

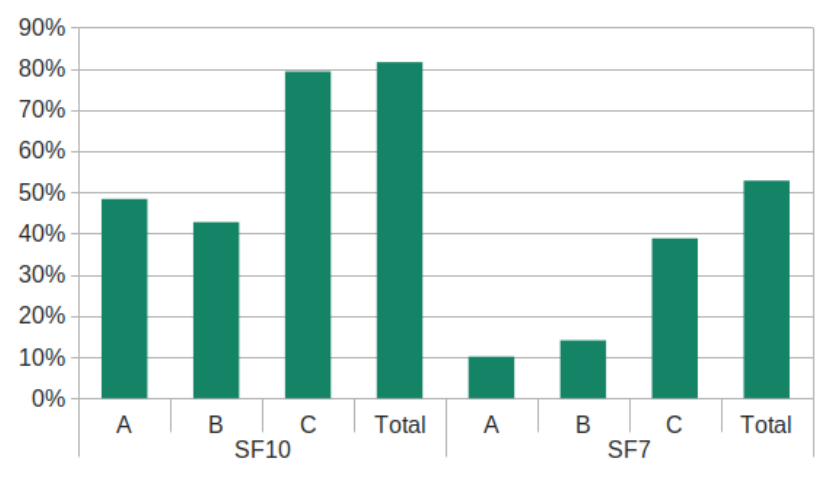

Figura 6: Taxa de recebimento de quadros enviados a partir do local 1. 


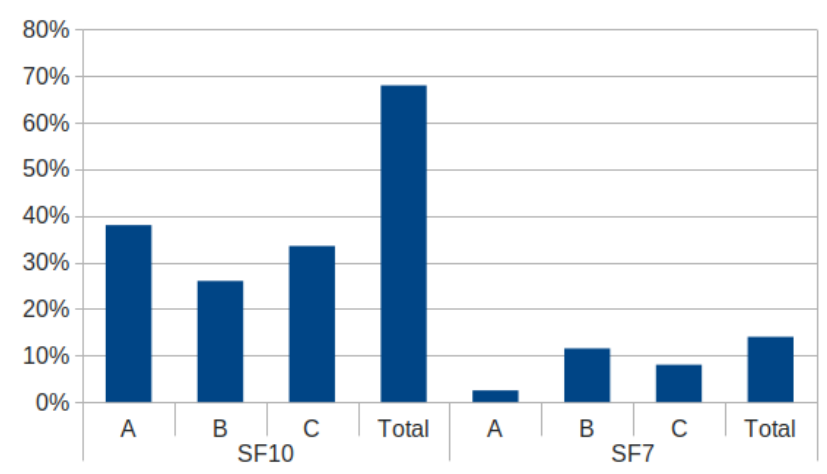

Figura 7: Taxa de recebimento de quadros enviados a partir do local 2.

\section{seria maior.}

Já no local 3 também havia a expectativa de comunicação apenas com o gateway B, conforme mostra a Fig. 4. Porém, como nos dois casos anteriores, os três gateways recebem dados do dispositivo. Neste teste, inclusive, fica claro que o percentual de recepção dos quadros pelo $\mathrm{ga}$ teway B fica limitado em função da limitação dos quatro canais. A taxa de recepção de quadros pelo dispositivo ficou próxima de $50 \%$ utilizando SF10 e SF7, conforme apresentado na Fig. 8.

Utilizando SF10, a taxa de recebimento dos quadros variou entre $36 \%$ e $57,5 \%$ nos gateways. O gateway B, apesar de ser o mais próximo, obteve uma taxa de recepção de $49 \%$. Considerando o somatório dos três gateways, a taxa de recebimento ficou em $82 \%$.

Ao alterar a configuração do dispositivo para SF7, somente $\mathrm{B}$ passa a receber os dados do dispositivo, conforme também demonstrado na Fig. 8. Dessa forma, mais uma vez, verifica-se que os gateways A e C são drasticamente afetados com a mudança do SF, enquanto B, por estar próximo, inclusive, aumenta a taxa de recepção para $49,5 \%$, reforçando, mais uma vez, que esse percentual está limitado pela quantidade de canais.

Por fim, no local 4 não foi possível o envio de quadros para nenhum dos gateways. Em relação ao gateway C, isso

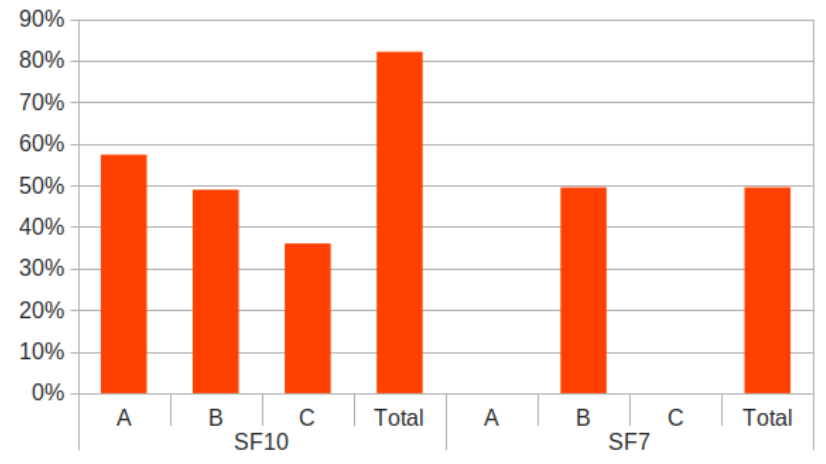

Figura 8: Taxa de recebimento de quadros enviados a partir do local 3. deveu-se ao fato do local estar localizado em um vale, tornando as montanhas localizadas à norte um obstáculo intransponível para o sinal LoraWAN. Em relação ao gateway B, além do motivo anterior, a maior distância e o fato deste estar localizado num local de alta densidade de edifícios atenuou o sinal de forma a também impedir a comunicação. Finalmente, em relação ao gateway C, soma-se aos argumentos o fato deste ser o gateway mais distante do local 4 .

De maneira geral, o local 1 foi o que apresentou os melhores resultados no que diz respeito à taxa de recepção de quadros, quanto à distância de transmissão. Em qualquer dos cenários avaliados, o dispositivo instalado no local conseguiu comunicar-se com todos os gateways, mesmo estando há quase $7 \mathrm{~km}$ distante do gateway $\mathrm{C}$, ou seja, mais que o dobro da distância estimada. Isso foi possível devidoà maior altitude e à baixa densidade de edifícios, oferecendo poucos obstáculos para comunicação. Por outro lado, o dispositivo instalado no local 4 apresentou os piores resultados, apesar de estar dentro da cobertura estimada de um dos gateways.

Esses resultados variados, inclusive, corroboram os apresentados no trabalho de Meireles et al. (2018). Isso demonstra que, apesar da tecnologia ser voltada à comunicação sem fio de longa distância com baixa potência, os obstáculos interferem significativamente na propagação do sinal, apresentando variações relevantes de cobertura conforme a densidade de edifícios ou a característica de relevo da região, demonstrando a importância de realização de estudos como esse para validar o funcionamento da tecnologia LoraWAN - e de qualquer outra tecnologia sem fio - em um ambiente real. No caso da cidade de Caxias do Sul, possivelmente seriam necessários mais dois desses equipamentos para atender a zona sudeste e a zona sudoeste da cidade, que possuem altitude mais baixa em relação ao restante da região.

Outro resultado relevante nos testes realizados referese à confiabilidade da rede. Mesmo no melhor cenário houve perda de $18,5 \%$ dos quadros transmitidos, conforme mostrado na Fig. 6. Isso demonstra que essa rede apresenta problemas de confiabilidade. Dessa forma, aplicações LoraWAN que necessitam dessa premissa devem habilitar o recurso de confirmação de recebimento de quadros disponível na subcamada MAC do protocolo, conforme tratado na Seção 2.2, ou implantar recursos de confirmação de recebimento de dados dentro de seu código, solicitando retransmissões de quadros que não foram recebidos corretamente.

\subsection{Avaliação conforme variação do payload do quadro e do SF utilizado na transmissão}

Conforme mostra a Fig. 9, percebe-se que, conforme o SF da transmissão é reduzido, taxa de recepção de quadros também é reduzida. Analisando o quadro com payload de 51 bytes, há uma queda abrupta na taxa de recepção de quadros quando alterado o SF de 10 para 9, onde apresentou uma queda de $60 \%$ para $48 \%$, mantendo-se praticamente estável nos demais SF.

Quando o quadro possui payload de 25 bytes, essa queda é observada quando o SF é alterado de 9 para 8, apresentado uma queda abrupta da taxa de recepção de $42 \%$ para $31 \%$. 


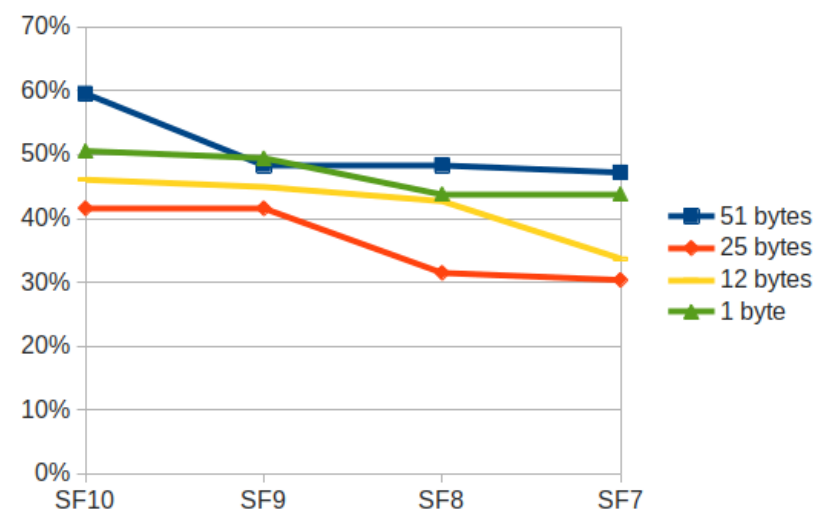

Figura 9: Taxa de recebimento de quadros conforme o tamanho, em bytes, do payload do quadro.

Nas demais alterações, a alteração não chega a apresentar alterações significativas.

Quando o quadro possui payload de 12 bytes, as alterações de SF10 para SF8 reduzem a taxa de recepção de $46 \%$ para 43\%, enquanto a alteração de SF8 para SF7 reduz a taxa de recebimento para apenas 34\%.

Por fim, quando o payload do quadro possui apenas 1 byte, não há grandes alterações na taxa de recepção de quadros, variando de $51 \%$ para $44 \%$. A maior alteração se dá na alteração do SF9 para SF8, onde a taxa de recebimento de quadros é reduzida de $49 \%$ para $44 \%$.

De maneira geral, essa queda na taxa de recepção nas reduções de SF era esperada pois a duração do chirp diminui conforme o SF é diminuído, conforme explanado na Seção 2. Todavia, conforme os resultados mostrados na Fig. 9, percebe-se que a carga de dados contida no payload do quadro interfere no momento onde essa queda ocorre de forma mais acentuada. Payloads perto do seu tamanho máximo tendem a apresentar uma queda maior na taxa de recepção na alteração do SF10 para o SF9, enquanto payloads com menos dados tendem a apresentar quedas maiores do SF8 para o SF7.

Além disso, a alteração do tamanho do payload do quadro não parece apresentar alterações significativas na taxa de recepção de quadros. Conforme também mostra a Fig. 9, a melhor taxa de recepção de quadros ocorreu nos quadros transmitidos com 51 bytes, seguidos dos quadros com 1 byte, 12 bytes e, finalmente, com 25 bytes.

O que mostra ter um maior impacto nessa taxa é o RSSI e o SNR da recepção, conforme apresentado na Fig. 10, que mostra os valores recebidos no gateway $\mathrm{B}$. Os quadros transmitidos a 1 byte tiveram o RSSI que variava entre -103 e -113, com média de $-108,3$, enquanto o SNR variava entre $5 \mathrm{~dB}$ e $-6 \mathrm{~dB}$, com média de $-0,4 \mathrm{~dB}$.

Os quadros transmitidos a 12 bytes tiveram o RSSI que variava entre -106 e -114, mas com média de -109,4, enquanto o SNR variava entre $3 \mathrm{~dB}$ e $-7 \mathrm{~dB}$, com média de $-1,2 \mathrm{~dB}$. Ou seja, no momento em que esses quadros foram transmitidos, apresentaram valores RSSI e SNR um pouco mais baixos em relação ao momento do envio dos quadros de 1 byte.

Já os quadros transmitidos a 25 bytes tiveram o RSSI

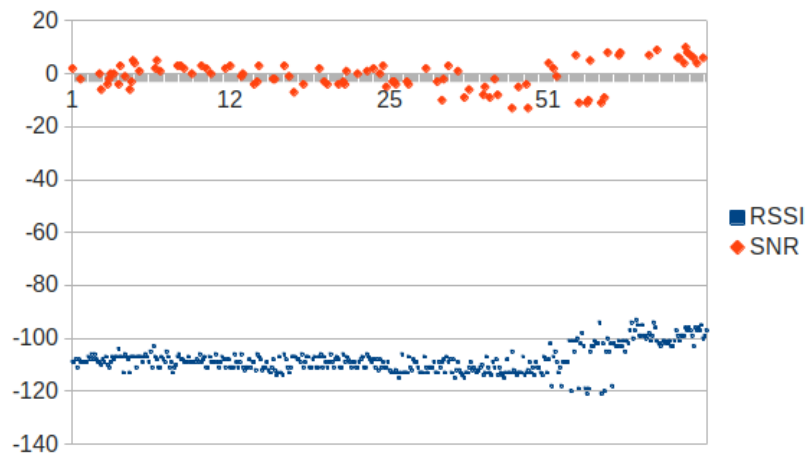

Figura 10: Valores de SNR, em dB, e RSSI registrados durante o recebimento dos quadros contendo 1, 12, 25 e 51 bytes.

que variava entre -105 e -115 , com média de $-111,4$, enquanto o SNR variava entre $3 \mathrm{~dB}$ e $-13 \mathrm{~dB}$, com média de $-4,9 \mathrm{~dB}$. Ou seja, no momento em que esses quadros foram transmitidos, apresentaram os menores valores de RSSI e SNR de todos os testes.

Finalmente, os quadros transmitidos a 51 bytes apresentaram a maior variação de RSSI, com valores entre -93 e -121 , mas com média de $-102,8$, enquanto o SNR variava entre $10 \mathrm{~dB}$ e $-11 \mathrm{~dB}$, com média de $2,5 \mathrm{~dB}$. Ou seja, apresentaram os maiores valores de SNR e RSSI e, consequentemente, as melhores taxas de transmissão de quadros.

\section{Conclusão}

Este trabalho apresentou os resultados do alcance e da confiabilidade da rede LoraWAN pública instalada na cidade de Caxias do Sul/RS, obtida através da taxa de recebimento de quadros. Essa medição considerou alterações no SF, no tamanho do payload e também diferentes locais onde a aplicação LoraWAN era executada. Verificou-se, durante o desenvolvimento desse trabalho, de que a configuração dos gateways estava incorretamente configurada para o padrão US902-928, ao invés do padrão AU915-928.

Durante a análise dos resultados, percebeu-se que a variação da altitude interferiu significativamente nessa taxa, enquanto o tamanho do payload do quadro não apresentou mudanças significativas nessa análise. Apesar da cobertura estimada dos gateways públicos ser superada na maior parte dos casos, em um desses locais o dispositivo LoraWAN não conseguiu transmitir para nenhum dos gateways instalados na cidade. Isso reforça a importância de trabalhos de avaliação como este para a correta avaliação e aplicação da tecnologia em um ambiente real, bem como a necessidade de instalação de mais desses equipamentos para atender toda a área urbana do município.

Além disso, no melhor cenário possível, a taxa de transmissão de quadros ficou em $81,5 \%$, o que demonstra que essa rede é recomendada para aplicações que não necessitem de transmissões de dados confiáveis. 


\section{Agradecimentos}

Agradecemos a todos que de alguma maneira contribuíram na elaboração deste trabalho, especialmente ao colega Mário Câmara, membro da comunidade TTN de Caxias do Sul.

\section{Referências}

Augustin, A., Yi, J., Clausen, T. and Townsley, W. M. (2016). A study of Lora: Long range \& low power networks for the internet of things, Sensors (Switzerland) 16(9): 1-18. http://dx.doi.org/10.3390/s16091466.

Barro, P. A., Zennaro, M. and Pietrosemoli, E. (2019). TLTN - The local things network: On the design of a LoRaWAN gateway with autonomous servers for disconnected communities, IFIP Wireless Days 2019-April: 2019-2022. http://dx.doi.org/10.1109/WD. 2019.8734239.

Benkahla, N., Tounsi, H., Song, Y. Q. and Frikha, M. (2021). Review and experimental evaluation of ADR enhancements for LoRaWAN networks, Telecommunication Systems . https://doi.org/10.1007/s11235-020-00738-x.

Caxias do Sul, P. M. (2014). Perfil socioeconômico de caxias do sul. Disponível em https : //cutt. ly/DzQ8834.

Chinato, A., Garcia, R., da Silva, J., Carvalho, E., de Lima, M., de Lima, J. V. and Ferrigo, S. (2020). Análise do funcionamento de dispositivos lora e lorawan na cidade de caxias do sul/rs, Anais da XX Escola Regional de Alto Desempenho da Região Sul, SBC, Porto Alegre, RS, Brasil, pp. 17-20. Disponível em https ://sol.sbc.org.br/ index.php/eradrs/article/view/10745.

Chinchilla-Romero, N., Navarro-Ortiz, J., Muñoz, P. and Ameigeiras, P. (2021). Collision avoidance resource allocation for LoRaWAN, Sensors (Switzerland) 21(4): 1-19. http://dx.doi.org/10.3390/s21041218.

Ertürk, M. A., Aydın, M. A., Büyükakkaşlar, M. T. and Evirgen, H. (2019). A Survey on LoRaWAN Architecture, Protocol and Technologies, Future Internet 11(10): 216. http://dx.doi.org/10.3390/fi11100216.

Ferrari, P., Sisinni, E., Carvalho, D. F., Depari, A., Signoretti, G., Silva, M., Silva, I. and Silva, D. (2020). On the use of LoRaWAN for the Internet of Intelligent Vehicles in Smart City scenarios, 2020 IEEE Sensors Applications Symposium, SAS 2020 - Proceedings . http: //dx. doi .org/ 10.1109/SAS48726.2020.9220069.

Ferrigo, S. F., da Costa, C. A. and da Rosa Righi, R. (2017). Um middleware para gerenciamento de sensores de saúde corporais em dispositivos móveis, Anais do IX Simpósio Brasileiro de Computação Ubíqua e Pervasiva, SBC. https://doi.org/10.5753/sbcup.2017.3310.

Gartner (2019). Gartner says 5.8 billion enterprise and automotive iot endpoints will be in use in 2020. Disponível em https://cutt. ly/wzQ8Hw1.

IBGE (2020). Ibge cidades. Disponível em https: //cidades.ibge.gov.br/brasil/rs/caxias-do-sul/ panorama.
Kufakunesu, R., Hancke, G. P. and Abu-Mahfouz, A. M. (2020). A survey on adaptive data rate optimization in lorawan: Recent solutions and major challenges, Sensors (Switzerland) 20(18): 1-25. http://dx . doi . org/10. 3390/s20185044.

LoRa, A. T. C. (2017). LoRaWAN 1.1 Specification, (1.1): 101. Disponível em https://lora-alliance.org/ resource-hub/lorawantm-specification-v11.

Marca, Y. and Scholze, S. (2015). Proposta de Substituição da Comunicação GSM em Smart Grids por Rádios de Longo Alcance, XXXIII Simpósio Brasileiro de Telecomunicações, SBrT, pp. 1-2. http://dx.doi.org/10.14209/ sbrt.2015.107.

Meireles, L., Garcia, L. and Segundo, A. (2018). Projeto e Implantação de Rede de Internet das Coisas em Regiões Montanhosas, XXXVI Simpósio Brasileiro de Telecomunicações e Processamento de Sinais, SBrT, pp. 16-19. https://doi.org/10.14209/sbrt.2018.253.

Network, T. T. (2020a). Frequency plans. Disponível em https://www. thethingsnetwork.org/docs/lorawan/ frequency-plans.html.

Network, T. T. (2020b). Frequency plans by country. Disponível em https://www.thethingsnetwork.org/docs/ lorawan/frequencies-by-country.html.

Network, T. T. (2020c). The things network. Disponível em https://www. thethingsnetwork.org/.

Noura, H., Hatoum, T., Salman, O., Yaacoub, J.-p. and Chehab, A. (2020). Internet of Things LoRaWAN security survey : Issues, threats and possible mitigation techniques, Internet of Things 12: 100303. https: //doi.org/10.1016/j.iot.2020.100303.

Ortiz, F. M., Cruz, P., Couto, R. d. S. and Costa, L. H. M. (2018). Caracterização de uma rede sem-fio de baixa potência e longo alcance para internet das coisas, Anais do XXXVI Simpósio Brasileiro de Redes de Computadores e Sistemas Distribuídos, SBC. Disponível em https://sol. sbc.org. br/index. php/sbrc/article/view/2485/2449.

Pioneiro, J. (2018). Caxias entra no mapa mundial da internet das coisas. Disponível em https : //cutt. ly/SzQ7TiM.

Polo, T. (2020). Trino polo: Polo de ti da serra gaúcha. Disponível em https ://www.trinopolo.com.br/.

Silva, J., Flor, D., Junior, V., Bezerra, N. and Medeiros, A. (2021). A Survey of LoRaWAN Simulation Tools in ns-3, Journal of Communication and Information Systems 36(1): 17-30. http://dx.doi.org/10.14209/jcis. 2021. 2.

Soares E Silva, F. E., Barriquello, C. H., Canha, L. N., Bernardon, D. P. and Seizo Hokama, W. (2018). Deployment of LoRA WAN Network for Rural Smart Grid in Brazil, Proceedings of the 2018 IEEE PES Transmission and Distribution Conference and Exhibition - Latin America, T and D-LA 2018 pp. 1-5. http: //dx. doi .org/10.1109/TDC-LA . 2018.8511646. 
Souza, A. T., Canha, L. N., Milbradt, R. G., Teixeira, C. M., Lemos, C. and Santana, T. A. S. (2019). XXV SNPTEE seminário nacional de produção e transmissão de energia elétrica.

Tanenbaum, A. S. (2003). Redes de Computadores, $4^{a}$ edição, Editora Campus.

Thieme, W. (2020). 5 wireless iot communication trends to watch in 2020. Disponível em https : //cutt. ly/jzQ5uJL.

Triantafyllou, A., Sarigiannidis, P., Lagkas, T., Moscholios, I. D. and Sarigiannidis, A. (2021). Leveraging fairness in LoRaWAN: A novel scheduling scheme for collision avoidance, Computer Networks 186(September 2020): 107735. https ://doi . org/10.1016/j . comnet . 2020.107735.

Villarim, M. R., De Luna, J. V. H., De Farias Medeiros, D., Pereira, R. I. S. and De Souza, C. P. (2019). LoRa performance assessment in dense urban and forest areas for environmental monitoring, INSCIT 2019 - 4th International Symposium on Instrumentation Systems, Circuits and Transducers pp. 1-5. http://dx.doi.org/10.1109/ INSCIT . 2019.8868567. 